Ohta, M. and A. Takahashi. Analysis of Nuclear Transmutation Induced from Metal Plus Multibody-FusionProducts Reaction. in Tenth International Conference on Cold Fusion. 2003. Cambridge, MA: LENRCANR.org. This paper was presented at the 10th International Conference on Cold Fusion. It may be different from the version published by World Scientific, Inc (2003) in the official Proceedings of the conference.

\title{
Analysis of Nuclear Transmutation Induced from Metal Plus Multibody-Fusion-Products Reaction
}

\author{
Masayuki Ohta and Akito Takahashi \\ Osaka University \\ Graduate School of Engineering, Department of Nuclear Engineering \\ Yamadaoka 2-1, Suita, Osaka, 565-0871, Japan \\ E-mail:mohta@newjapan.nucl.eng.osaka-u.ac.jp
}

\begin{abstract}
Nuclear transmutation is analyzed by the selective channel scission model. The fission product yields for Pd plus $\alpha$ or ${ }^{8} \mathrm{Be}$ reactions are calculated as secondary reactions of the multi-body fusion. And an anomalous isotopic ratio of Fe, which is reported by many researchers, is also analyzed and the analytical result shows good consistency with experimental results.
\end{abstract}

\section{Introduction}

Nuclear transmutation has been reported by lots of experiments. The features of this phenomenon are the production of elements which shows fission-like distribution and their anomalous isotopic ratios or the selective $\mathrm{Z}$ and mass changes [1]. To analyze this phenomenon, the selective channel scission (SCS) model has been proposed and improved [2]. The SCS model is powerful and can predict the fission product yields of any elements' fission.

The fission product yields are calculated as follows. At first, we consider all fission channels, which are possible combinations of atomic and mass numbers. The value of channel-dependent fission barrier $E_{\mathrm{f}}$ is estimated by the Q-value of the fission channel and the bare Coulomb potential $E_{\mathrm{c}}$ at the effective scission distance $R_{\text {eff. }}$ The $R_{\text {eff }}$ is obtained by setting the jointing factor $\eta$ which reflects the jointing force of strong interaction: $R_{\text {eff }}=\eta\left(R_{1}\right.$ $+R_{2}$ ), where $R_{1}$ and $R_{2}$ are the radiuses of the fission fragments. For the channel, the tunnel fission probability, that is the channel-opening probability, is calculated. If the nuclear excitation energy $E_{\mathrm{x}}$ is less than $E_{\mathrm{f}}$, the tunnel fission probability needs to be considered. On the other hand, this value is 1 if the $E_{\mathrm{x}}$ is more than $E_{\mathrm{f}}$. Then, the fission product yield is obtained by summing up these probabilities all over fission channels. These processes of the calculation are introduced in the previous paper in detail [2].

We have analyzed the nuclear transmutation, assuming photo-fission of cathode material, such as $\mathrm{Pd}$, excited by multi-photon process. This time, the nuclear transmutation is analyzed as fissions from secondary reactions of multi-body fusion [3] in addition to photo-fission.

\section{Models}

In general, nuclear transmutations have two types, roughly speaking. One has fission-like features of the cathode material. The other is the production of a little heavier elements than the cathode material, for example the production of $\mathrm{Cd}$ and $\mathrm{Sn}$ for Pd-type experiment [4]. 
The latter contains the mass- 8 and Z-4 increased transmutation reported by Y. Iwamura, for example from Cs to $\operatorname{Pr}[1]$.

We have analyzed the former, assuming photo-fissions of the cathode elements on the multi-photon induced fission (MPIF) model. The X-ray productions have been reported in some experiments. If these are burst-like productions, the flux is very high and the nuclei can be excited by these photons through interferences such as Rayleigh scattering and Compton scattering. Also, we have considered the production of photons from multi-body fusion reactions as the photon source. These photon emissions can be the source which induces MPIF in the experiments. The essence of MPIF is the collective excitation by these many low energy photons to $\mathrm{E} 1$ giant resonance region to make fissions.

We have calculated the fission product yields by MPIF and the results showed good agreement with the experimental results [2].

But the latter type of nuclear transmutations, which cannot be explained by fission, should be also explained in addition to the fission-like type. The fusions as secondary reactions of multi-body fusion may be occurred and the fissions could be induced for the excited nuclei.

Multi-body fusion model [3] has proposed to explain the anomalous ${ }^{4} \mathrm{He}$ production, which cannot be explained by usual DD fusion reaction. This model assumes that the multi-body fusion reaction rate is enhanced in the transient process by the coherent motion of hydrogen isotopes occupied octahedral or tetrahedral sites in Pd lattice. It can be considered that the symmetric properties of hydrogen isotopes in Pd lattice realize the situation inducing multibody fusion reactions.

The strong electron-screening effect may be induced by electron-phonon interactions in Pd deuterides. It is known that for high loading ratio of $\mathrm{PdH}_{\mathrm{x}}$ or $\mathrm{PdD}_{\mathrm{x}}$, the electron-phonon interaction becomes strong, and superconductivity arises [5]. In particular, the optical mode contributes to the electron-phonon interaction [6]. The electron-phonon interaction, that is Cooper pair-like coupling under the transient condition of multi-body fusion, enhances the fusion rates, in particular for 4D and 8D fusion reactions, in Pd deuterides. These considerations were reported in another paper in more detail $[7,8]$.

$$
\begin{aligned}
& 4 \mathrm{D} \rightarrow{ }^{8} \mathrm{Be}^{*} \rightarrow 2{ }^{4} \mathrm{He}+47.6 \mathrm{MeV}, \\
& 8 \mathrm{D} \rightarrow{ }^{16} \mathrm{O}^{*} \rightarrow 2{ }^{8} \mathrm{Be}+95.2 \mathrm{MeV}
\end{aligned}
$$

The latter transmutations are probably related to ${ }^{8} \mathrm{Be}$ or ${ }^{4} \mathrm{He}$ produced by these multi-body fusion reactions, judging from the increase in mass or $\mathrm{Z}$ with a specific number.

If the $8 \mathrm{D}$ fusion reaction occurs, that induces the mass- 8 and Z-4 increased transmutation and ${ }^{4} \mathrm{He}$ production, and further, that the excited nucleus may make fission. The same analogy can be applied to the case of 4D fusion reaction.

$$
\begin{gathered}
\mathrm{M}(\mathrm{Z}, \mathrm{A})+{ }^{4} \mathrm{He} \rightarrow \mathrm{M}(\mathrm{Z}+2, \mathrm{~A}+4), \\
\mathrm{M}(\mathrm{Z}, \mathrm{A})+{ }^{8} \mathrm{Be} \rightarrow \mathrm{M}(\mathrm{Z}+4, \mathrm{~A}+8), \\
\mathrm{M}(\mathrm{Z}, \mathrm{A})+{ }^{4} \mathrm{He}\left(\text { or }^{8} \mathrm{Be}\right) \rightarrow \text { Fission product } 1+\text { Fission product 2, }
\end{gathered}
$$

where $\mathrm{M}(\mathrm{Z}, \mathrm{A})$ shows the element with atomic number $\mathrm{Z}$ and mass number $\mathrm{A}$ in the sample. 


\section{Analytical results}

\subsection{General}

Fissions can occur as secondary reactions of multi-body fusion reactions that the sample metal Pd plus major products, that is ${ }^{4} \mathrm{He}$ or ${ }^{8} \mathrm{Be}$. This is because these excitation energies are enough high to induce fission reactions.

$\mathrm{Pd}$ has six isotopes in nature, and the natural abundances are listed in Table 1. And the compound nuclei for those plus ${ }^{4} \mathrm{He}$ and ${ }^{8} \mathrm{Be}$ reactions and the excitation energies are listed altogether, respectively.

Table 1. Natural abundance of Pd isotopes and excitation energies of compound nucleus by $+\alpha$ and $+{ }^{8} \mathrm{Be}$ reactions.

\begin{tabular}{|l|l||l|l|l|l|}
\hline Nuclides & $\begin{array}{l}\text { Natural } \\
\text { abundance (\%) }\end{array}$ & $\begin{array}{c}+\alpha(23.8 \\
\mathrm{MeV})\end{array}$ & $\begin{array}{l}\text { Excitation } \\
\text { energy (MeV) }\end{array}$ & $\begin{array}{l}+{ }^{8} \mathrm{Be}(47.6 \\
\mathrm{MeV})\end{array}$ & $\begin{array}{l}\text { Excitation } \\
\text { energy } \\
(\mathrm{MeV})\end{array}$ \\
\hline \hline${ }^{102} \mathrm{Pd}$ & 1.02 & ${ }^{106} \mathrm{Cd}^{*}$ & 25.4 & ${ }^{110} \mathrm{Sn}^{*}$ & 50.4 \\
\hline${ }^{104} \mathrm{Pd}$ & 11.14 & ${ }^{108} \mathrm{Cd}^{*}$ & 26.1 & ${ }^{112} \mathrm{Sn}^{*}$ & 51.8 \\
\hline${ }^{105} \mathrm{Pd}$ & 22.33 & ${ }^{109} \mathrm{Cd}^{*}$ & 26.3 & ${ }^{113} \mathrm{Sn}^{*}$ & 52.5 \\
\hline${ }^{106} \mathrm{Pd}$ & 27.33 & ${ }^{110} \mathrm{Cd}^{*}$ & 26.7 & ${ }^{114} \mathrm{Sn}^{*}$ & 53.2 \\
\hline${ }^{108} \mathrm{Pd}$ & 26.46 & ${ }^{112} \mathrm{Cd}^{*}$ & 27.3 & ${ }^{116} \mathrm{Sn}^{*}$ & 54.5 \\
\hline${ }^{110} \mathrm{Pd}$ & 11.72 & ${ }^{114} \mathrm{Cd}^{*}$ & 27.9 & ${ }^{118} \mathrm{Sn}^{*}$ & 55.8 \\
\hline
\end{tabular}

\section{2 $P d+\alpha$ reaction}

The jointing factor $\eta$ was taken as 1.84 for all Cd's isotopes in Table 1. The fission barriers for each isotopes of $\mathrm{Cd}$, which is the compound nucleus of $\mathrm{Pd}+\alpha$ reaction, were calculated. Then, the channel opening probabilities of channels for their excitation energies were calculated and we can obtain the yield data of fission products with considerations of the isotopic abundances.

The Z-distribution of fission product yields of $\mathrm{Pd}+{ }^{4} \mathrm{He}$ reaction calculated by SCS model is shown in Fig. 1-(a). The experimental result is the data by T. Mizuno, which is the same in the analysis by $\mathrm{Pd}$ photo-fission [2]. In this analysis, $\mathrm{Fe}$, Ti and $\mathrm{Cr}$ are most dominant products. The fission product yields at right and left wings are slightly larger than the Mizuno's experimental data. But the main part keeps good agreements with the Mizuno's data in rough approximation. The effect of nuclear shell structure may appear, especially about alpha-clustered nuclei.

And the mass-distribution of fission products from $\mathrm{Pd}+{ }^{4} \mathrm{He}$ reaction is obtained as shown in Fig. 1-(b). Overall, the feature of mass-distribution corresponds to Z-distribution. We can say that a qualitative agreement with Mizuno's experimental data is obtained in the main part.

\section{3 $\mathrm{Pd}+{ }^{8}$ Be reaction}

Next, the fission product yields from $\mathrm{Pd}+{ }^{8} \mathrm{Be}$ reaction were calculated. The jointing factor $\eta$ was taken as 1.20 for all Sn's isotopes.

The Z-distribution of fission product yields was shown in Fig. 2-(a). As well as the case of $\mathrm{Pd}+{ }^{4} \mathrm{He}$ reaction, the fission products in the center part are $\mathrm{Fe}, \mathrm{Ti}, \mathrm{Cr}$ and so on. The fission product yields at right and left wings increase because of the distributions of their fission barriers. 
And the mass-distribution of fission products from $\mathrm{Pd}+{ }^{8} \mathrm{Be}$ reaction was shown in Fig. 2(b). Right and left parts are higher than the experimental results as well as those in Zdistribution.

\subsection{Isotopic abundance}

It is also known that the nuclear transmutation products show the anomalous isotopic ratios, which were different from natural abundance. And that is the strong evidence to suggest that some nuclear reactions should occur in the experiments.

Figure 3 shows the comparison of isotopic ratio of Fe with some experimental results. Starting from the left, the natural abundance, experimental results by Mizuno [2], Iwamura [1], Karabut [4] and the analytical results by MPIF (photo-fission) [2], $\mathrm{Pd}+{ }^{4} \mathrm{He}$ and $\mathrm{Pd}+{ }^{8} \mathrm{Be}$ reactions.

In particular, the feature of analytical results is the high ratio of ${ }^{58} \mathrm{Fe}$. It may be possible to explain the extraordinary isotopic ratio of the experimental results which show differences from the natural abundance.

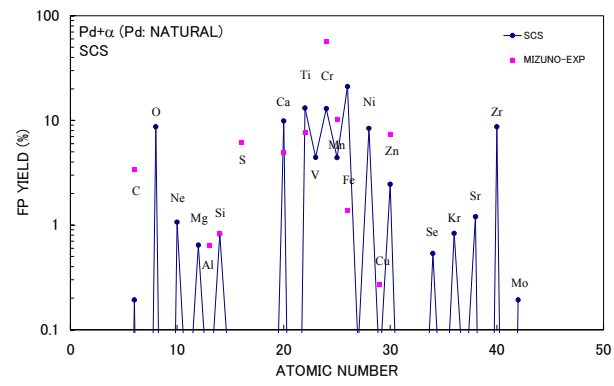

(a)

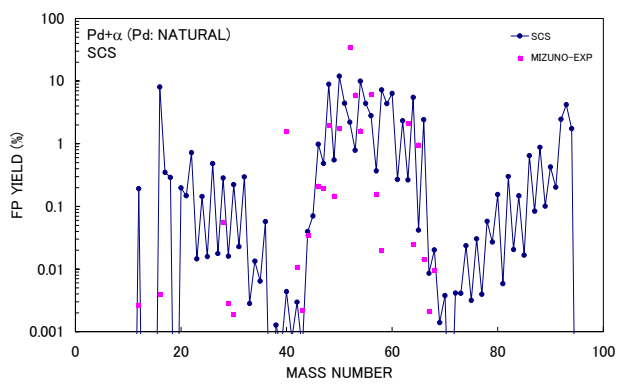

(b)

Figure 1. Fission Product Yield for Pd $+\alpha$ reaction: (a) Z-number and (b) Mass-number.

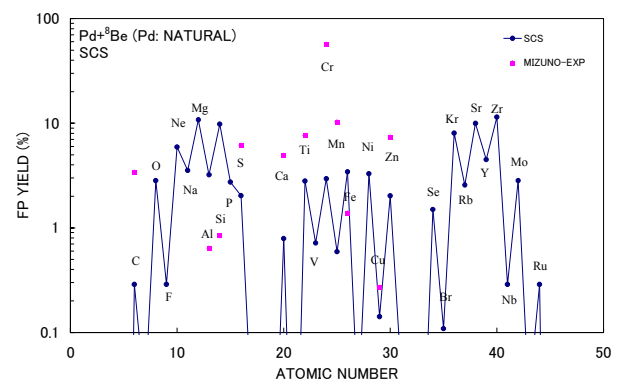

(a)

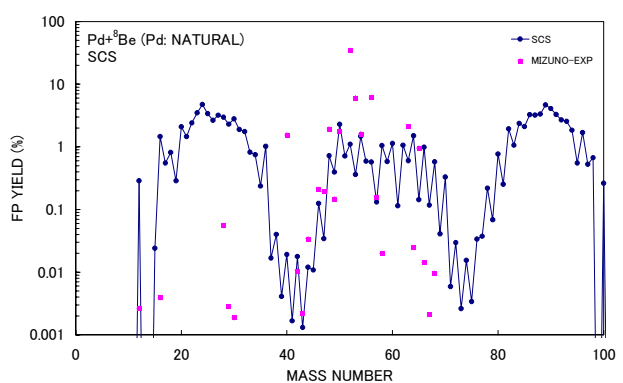

(b)

Figure 2. Fission Product Yield for $\mathrm{Pd}+{ }^{8} \mathrm{Be}$ reaction: (a) Z-number and (b) Mass-number. 


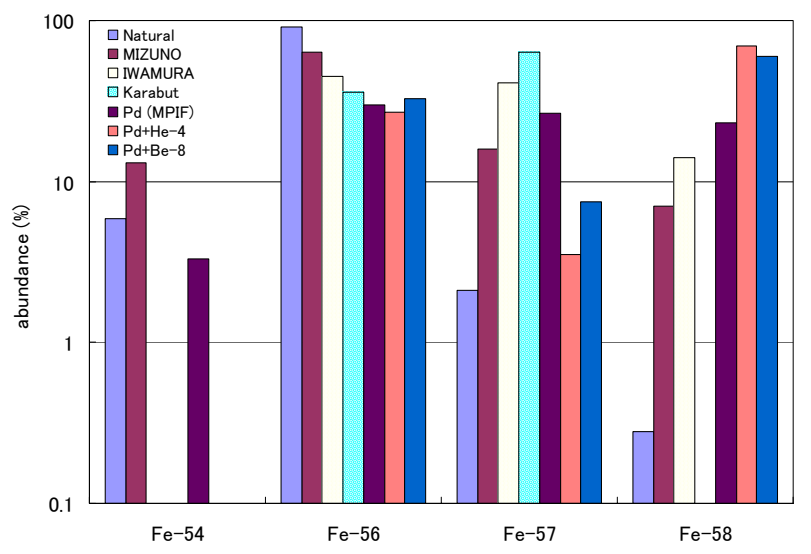

Figure 3. Comparison of isotopic ratios between natural Fe, SCS analysis and experiments.

\section{Discussion and Conclusion}

Nuclear transmutation was analyzed by SCS model and the analytical fission product yields were obtained for processes of $\mathrm{Pd}+{ }^{4} \mathrm{He}$ and $\mathrm{Pd}+{ }^{8} \mathrm{Be}$ in addition to the photo-fission of $\mathrm{Pd}$ in the previous paper [2].

It is also worth noting that $\mathrm{Cd}$ and $\mathrm{Sn}$ were detected simultaneously in some of experiments [4] in addition to fission-like elements. The Z-numbers of these elements are two or four larger than Pd. Also, the transmutations from $\mathrm{Cs}$ to $\mathrm{Pr}$ and from $\mathrm{Sr}$ to Mo were reported in Mitsubishi-type experiments [1]. These are mass- 8 and Z-4 increased type transmutations. So ${ }^{4} \mathrm{He}$ or ${ }^{8} \mathrm{Be}$ must induce these type transmutations as a secondary reaction. This type of nuclear transmutation is explained by the formation of compound nuclei from multi-body fusion reaction.

The another-type of nuclear transmutation which produces lighter elements will probably be explained by fission. We have analyzed by SCS model, assuming three processes of photo-fission [2], $\mathrm{Pd}+{ }^{4} \mathrm{He}$ and $\mathrm{Pd}+{ }^{8} \mathrm{Be}$ reactions. It is assumed that these processes occur independently. We cannot decide which kind of fission is suitable and dominant only in this analysis. Actually, these processes will occur simultaneously. It should be estimated by the composition of processes of $\mathrm{Pd}+{ }^{4} \mathrm{He}$ and $\mathrm{Pd}+{ }^{8} \mathrm{Be}$ reactions, even and the photo-fission of Pd.

For more detailed analyses, the fission-mode should be considered in the calculation processes. This is the fine adjustment of the calculations. We have already analyzed the neutron-induced fission for U-235 and it showed good agreements with the data [9].

Another task is the analysis of $\gamma$-ray emission. It is possible to estimate it by following the decay processes in the analytical results in detail.

\section{References}

1. Y. Iwamura et al., Jpn. J. Appl. Phys., 41, 4642 (2002).

2. A. Takahashi et al., Proc. ICCF8, 397 (2000); and A. Takahashi et al., Jpn. J. Appl. Phys., 40, 7031 (2001).

3. A. Takahashi et al., Fusion Technol., 27, 71 (1995). 
4. A.B. Karabut, Proc. ICCF9, 151 (2002).

5. R.W. Standley et al., Solid State Commun., 31, 801 (1979).

6. J.P. Burger, Metal Hydrides, G. Bambakidis ed. (Plenum, 1981), p. 243.

7. M. Ohta et al., Proc. ICCF8, 403 (2000).

8. A. Takahashi, Proc. JCF4, 74 (2002); and Proc. ICCF10 (this issue).

9. M. Ohta et al., Jpn. J. Appl. Phys., 42, 645 (2003). 